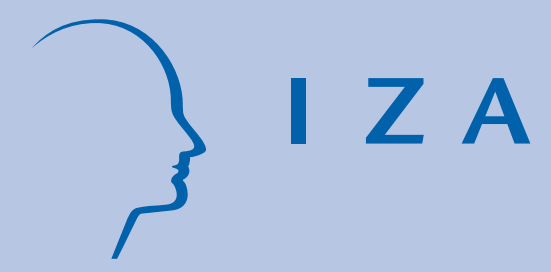

IZA Standpunkte Nr. 49

Effizient, einfach und gerecht:

Ein integriertes System zur Reform von

Einkommensteuer und Sozialabgaben

Max Löffler Hilmar Schneider

Andreas Peichl Sebastian Siegloch

Nico Pestel

Juli 2012 


\title{
Effizient, einfach und gerecht: Ein integriertes System zur Reform von Einkommensteuer und Sozialabgaben
}

\author{
Max Löffler \\ IZA und Universität zu Köln \\ Andreas Peichl \\ IZA, Universität zu Köln, ISER und CESifo \\ Nico Pestel \\ IZA und Universität zu Köln \\ Hilmar Schneider \\ IZA und DIW Berlin \\ Sebastian Siegloch \\ IZA und Universität zu Köln
}

IZA Standpunkte Nr. 49

Juli 2012

IZA

Postfach 7240

53072 Bonn

Tel.: (0228) 3894-0

Fax: (0228) 3894-180

E-Mail: iza@iza.org

Die Schriftenreihe "IZA Standpunkte" veröffentlicht politikrelevante Forschungsarbeiten und Diskussionsbeiträge von IZA-Wissenschaftlern, IZA Research Fellows und IZA Research Affiliates in deutscher Sprache. Die Autoren sind für den Inhalt der publizierten Arbeiten verantwortlich. Im Interesse einer einheitlichen Textzirkulation werden Aktualisierungen einmal publizierter Arbeiten nicht an dieser Stelle vorgenommen, sondern sind gegebenenfalls nur über die Autoren selbst erhältlich. 


\title{
ZUSAMMENFASSUNG
}

\section{Effizient, einfach und gerecht: Ein integriertes System zur Reform von Einkommensteuer und Sozialabgaben}

Das deutsche Einkommenssteuerrecht ist zu komplex und zu intransparent. Zahlreiche Reformkonzepte wurden in den vergangenen Jahren öffentlich diskutiert. Allerdings ist es bisher nicht gelungen, den bestehenden Zielkonflikt zu überwinden und positive Beschäftigungsund Verteilungswirkungen bei einem neutralen Effekt auf den Staatshaushalt zu erzielen. Ein Problem der bisher gemachten Vorschläge ist, dass sie die Sozialversicherungsabgaben und deren Interaktion mit der Einkommensteuer vernachlässigen. Unser Reformvorschlag setzt genau an dieser Stelle an und schlägt ein integriertes Steuer- und Abgabensystem vor. Unsere Simulationsstudie zeigt, dass durch diese umfassende Reform der beschriebene Zielkonflikt überwunden werden kann: Die Beschäftigung steigt, die Einkommensungleichheit sinkt und die Steuereinnahmen steigen leicht.

\begin{abstract}
The system of income taxation in Germany is too complex and too intransparent. Many reform proposals have been publicly debated during the past years. However, so far, conflicting goals of positive employment und distributional effects without a negative impact on the state's budget could not be overcome. A common problem of previous reform proposals is that they have neglected social security contributions and their interactions with income taxes. We tackle this issue by proposing an integrated system of taxation. Our simulations show that this comprehensive reform resolves conflicting goals: employment increases, inequality decreases and tax revenues slightly increase.
\end{abstract}

JEL-Codes: D31, H24, J21

Schlagworte: Steuervereinfachung, Steuerreform, Sozialversicherung, Mikrosimulation, Arbeitsangebot, Einkommensungleichheit

Kontaktadresse:

Andreas Peichl

IZA

Postfach 7240

D-53072 Bonn

E-Mail: peichl@iza.org 


\section{Einleitung}

Das deutsche Einkommensteuerrecht wird seit Jahren als zu wenig transparent und als zu komplex kritisiert. Ebenso in der Kritik steht der im internationalen Vergleich sehr hohe Steuer- und Abgabenkeil insbesondere für mittlere Einkommen (,Mittelstandsbauch“, siehe auch OECD, 2011). In der steuerpolitischen Diskussion wurden deshalb immer wieder Vorschläge für mögliche Reformen des Steuer- und Abgabensystems gemacht. Besondere Aufmerksamkeit hat dabei der Tarifverlauf der Einkommensteuer erhalten, denn im Gegensatz zu seinen europäischen Nachbarn wendet nur Deutschland eine mehrstufige Progressionsformel mit stetig steigendem Grenzsteuersatz an. Als Alternative zum linear-progressiven Tarifverlauf wurde beispielsweise von Paul Kirchhof $(2003,2011)$ ein einheitlicher Grenzsteuersatz vorgeschlagen. Andere Konzepte sehen verschiedene Stufen-Tarife vor (z.B. FDP, 2008, 2009, oder Rose, 2011). Simulationsstudien dieser Reformvorschläge zeigen jedoch, dass es keinem der Konzepte gelingt, den existierenden Zielkonflikt zu überwinden. In keinem Fall konnten positive Beschäftigungs- und Verteilungswirkungen bei gleichzeitig neutralem Aufkommenseffekt erzielt werden. ${ }^{1}$

Den erwähnten Vorschlägen ist gemein, dass sie sich auf eine Reform der Einkommensteuer beschränken und das Zusammenwirken zwischen Sozialversicherung und Einkommensteuer vernachlässigen. ${ }^{2}$ Insbesondere für kleine und mittlere Einkommen spielen aber die Sozialversicherungsbeiträge eine erhebliche

\footnotetext{
${ }^{1}$ Siehe z.B. Bach et al. (2004), Bach (2005), Fuest et al. (2007a, b), Neumann et al. (2009), aus dem Moore et al. (2009, 2010), Peichl et al. (2010, 2011), Houben et al. (2011) und Löffler et al. (2012).

${ }^{2}$ Eine Ausnahme stellt das Gutachten von Bach et al. (2002) dar. Darin wird eine teilweise Steuerfinanzierung der Sozialversicherung diskutiert und deren Budget- und Beschäftigungswirkungen grob abgeschätzt. Im Gegensatz dazu schlagen wir ein deutlich umfassenderes Reformkonzept vor und analysieren die Auswirkungen auf Grundlage von detaillierten Mikrodaten sowie eines mikroökonometrischen Arbeitsangebots- und Arbeitsnachfragemodells.
} 
Rolle. So betrug 2007 der Anteil der direkten Steuern am BIP rund 11\%, während der Anteil der Sozialversicherungsbeiträge bei mehr als 16\% lag (vgl. OECD, 2011). Zielt eine Reform also auf stärkere Erwerbsanreize oder eine Reduzierung der Einkommensungleichheit ab, dann sollte die Rolle der Sozialversicherungsabgaben nicht unterschätzt werden. ${ }^{3}$ Während im EU- oder OECD-Durchschnitt die Sozialabgaben maximal die gleiche Bedeutung wie die Einkommensteuer haben, legt Deutschland ein höheres Gewicht auf Sozialversicherungsbeiträge - nur Frankreich weist hier einen noch höheren Anteil auf (vgl. OECD, 2011). Aufgrund von Beitragsbemessungs- und Pflichtversicherungsgrenzen wirken die Sozialabgaben in Deutschland jedoch sehr stark regressiv und kompensieren somit (teilweise) die hohe effektive Progression der deutschen Einkommensteuer. ${ }^{4}$ In der Vergangenheit wurde bereits des Öfteren eine progressive Ausgestaltung der Sozialversicherungsbeiträge insbesondere im unteren Einkommensbereich - vorgeschlagen. Solche Vorschläge leiden jedoch unter den bekannten Anreiz- und Finanzierungsproblemen von Kombilöhnen (Wissenschaftlicher Beirat beim Bundesministerium der Finanzen, 2008), da die Interaktionen mit steuerlichen Regelungen (wie z.B. dem Ehegattensplitting) unberücksichtigt bleiben.

Im vorliegenden Beitrag skizzieren wir ein integriertes Steuer- und Abgabensystem, das die Regressivität der Sozialversicherungsabgaben beseitigt und folglich eine

\footnotetext{
${ }^{3}$ Arnold et al. (2011) zeigen, dass es im Hinblick auf Wirtschaftswachstum zwar prinzipiell besser ist, den Steuermix von direkten auf indirekte Steuern umzuschichten. Wenn dies aber insbesondere aus verteilungspolitischen Gesichtspunkten nicht wünschenswert ist, zeigt sich, dass eine Entlastung niedriger bis mittlerer Einkommen einer Entlastung höherer Einkommen auch aus wachstums- und beschäftigungspolitischen Gesichtspunkten vorzuziehen ist.

${ }^{4}$ Vgl. Peichl und Schaefer (2009). So zahlen die obersten 10(5)[1]\% der Steuerpflichtigen mehr als 50(40)[20]\% des Einkommensteueraufkommens in Deutschland, wohingegen von den unteren 50\% der Steuerpflichtigen nur rund 5\% des Steueraufkommens stammen. Dagegen ist die prozentuale Belastung mit SV-Beiträgen in der Mitte der Einkommensverteilung höher (vgl. RWI/FiFo, 2007). So zahlen beispielsweise die obersten 10\% der Einkommensverteilung rund 23\% der gesamnten SV-Beiträge während die untersten $50 \%$ mehr als $16 \%$ beisteuern (vgl. RWI/FiFo, 2009, RWI, 2011, detailliertere Zahlen für die oberen $1 \%$ bzw. $5 \%$ sind nicht verfügbar).
} 
gezielte Reduktion der starken Progression im Bereich der mittleren Einkommen ermöglicht. Dieser Reformvorschlag greift die genannten Kritikpunkte auf und verbindet die Ziele Beschäftigungszuwachs und, Reduktion der Ungleichheit unter Wahrung fiskalischer Neutralität. Eine solche Reform wäre ein wichtiger Schritt, um das Steuer- und Abgabensystem weniger anfällig für die Auswirkungen der stark alternden Bevölkerung zu machen. Unsere Simulationsrechnungen zeigen, dass das Erreichen aller drei Ziele sehr wohl möglich ist, dafür jedoch umfassende Änderungen am gesamten Steuer- und Abgabensystem notwendig wären. Eine reine Reform des Tarifverlaufs der Einkommensteuer reicht hierfür nicht aus. Kernstück unseres Vorschlags ist die Überführung der beitragsfinanzierten Sozialversicherung in ein steuerfinanziertes Sozialsystem, um so ein einheitliches und integriertes System für Umverteilung und Aufkommenserzielung zu schaffen. Die Einkommensteuer wird zudem deutlich vereinfacht. Hier greifen wir die Vorschläge von Kirchhof (2003, 2011) auf und gehen mit einer noch umfassenderen und breiteren Definition der steuerlichen Bemessungsgrundlage einen Schritt weiter. An die Stelle der Tarifformel tritt ein progressiver Fünf-Stufen-Tarif, dessen Grenzsteuersätze die bisherigen Sozialversicherungsbeiträge sowie den Solidaritätszuschlag mit abdecken. Auch wenn die Umsetzung der Gesamtreform kurzfristig wenig realistisch erscheint, sollten sich zukünftige Reformschritte zur Vereinfachung des Steuer- und Abgabensystems an diesen Punkten orientieren, um negative Wirkungen auf die Einkommensverteilung, die Beschäftigung und die öffentlichen Haushalte zu vermeiden.

Der Beitrag ist weiter wie folgt strukturiert. Abschnitt 2 skizziert die von uns vorgeschlagenen Reformvarianten. Abschnitt 3 stellt Methodik und Datenbasis vor. In 
Abschnitt 4 folgt die Präsentation der Simulationsergebnisse. Der Beitrag schließt mit einem Fazit in Abschnitt 5.

\section{Reformvorschlag: Integriertes Steuer- und Abgabensystem}

Unser Reformvorschlag für ein integriertes Steuer- und Abgabensystem besteht aus einer umfassenden Vereinfachung, wobei ein Kernstück die Steuerfinanzierung der Sozialsysteme ist. Die Ansprüche im Transferbereich (Arbeitslosengeld II, Kindergeld, Kinderzuschlag und Wohngeld) bleiben unberührt. Die vorgeschlagenen Änderungen gegenüber dem aktuellen Rechtsstand (November 2011) lassen sich in drei Bereiche unterteilen und stellen sich wie folgt dar.

\section{Reform der Sozialversicherungen}

Ziel einer Reform des Sozialversicherungssystems muss es sein, die nachhaltige Finanzierung zu gewährleisten, ohne den Solidarausgleich zu vernachlässigen. In Deutschland erfolgt bislang eine Einkommensumverteilung sowohl innerhalb des staatlichen Steuer- und Transfersystems als auch innerhalb der Sozialversicherungen. Dies führt zu unnötigen Transaktionskosten und zusätzlicher Intransparenz. Insbesondere mittlere Einkommensbereiche werden überproportional belastet. Umverteilungsmaßnahmen und versicherungsfremde Leistungen sollten daher ausschließlich über Steuern finanziert werden. Deshalb werden die bisher überwiegend beitragsfinanzierten Sozialversicherungen in ein steuerfinanziertes Sozialsystem überführt, das eine Garantierente und eine Grundversorgung vergleichbar mit den Leistungen der heutigen Arbeitslosen-, Kranken- und Pflegeversicherung bereitstellt. Durch die Zusammenführung von Sozialabgaben und 
Einkommensteuer werden die Regelungen zur geringfügigen Beschäftigung und zur Gleitzone überflüssig.

Mit dem Wegfall der Beitragsfinanzierung entfällt auch der Arbeitgeberanteil zur Sozialversicherung. Stattdessen müssen die Arbeitgeber jedoch in gleicher Höhe eine Lohnsummensteuer als Aufschlag zu den Unternehmenssteuern zahlen. Der hier präsentierte Reformvorschlag führt deshalb nicht direkt zu einer Entlastung der Unternehmen. Gleichwohl bringt er durch die Zusammenführung von Lohnsteuer und Sozialversicherung sowie durch die Vereinfachung der Bemessungsgrundlage eine Reduktion des bürokratischen Aufwands mit sich. Wir nehmen im Folgenden an, dass das Aufkommen aus der Lohnsummensteuer der Summe der heutigen Arbeitgeberbeiträge zur Sozialversicherung entspricht. Die Bruttoarbeitskosten ändern sich somit für die Arbeitgeber nicht. Damit wird von direkten Wirkungen auf die Arbeitsnachfrage abstrahiert.

\section{Reform der Bemessungsgrundlage}

Die Berechnung des zu versteuernden Einkommens - der Bemessungsgrundlage für die neue Einkommensteuer - wird in unserem Reformvorschlag stark vereinfacht, indem die Gesamtsumme aller Bruttoeinkommen zur Steuer herangezogen wird und die Abzugsmöglichkeiten ähnlich wie von Kirchhof (2011) vorgesehen erheblich eingeschränkt werden. Durch die Abschaffung der Sozialversicherungen (SV) entfällt auch der Abzug von SV-Beiträgen, der die Bemessungsgrundlage heute deutlich schmälert. Sämtliche bestehenden Abzüge bis auf den Werbungskosten- und den Sparerpauschbetrag entfallen. Die Werbungskostenpauschale für Einkommen aus nichtselbstständiger Arbeit wird auf 2.000 Euro angehoben (vgl. Kirchhof, 2011). Auch die Günstigerprüfung zwischen Kinderfreibetrag und Kindergeld entfällt, da das 
Kindergeld als einheitliche einkommensunabhängige Steuergutschrift gewährt wird. ${ }^{5}$

Unser Vorschlag sieht zudem eine breitere Definition der Bruttoeinkommen vor. So werden Einkommen aus Kapital wieder als gewöhnliche Einkommensart zur Einkommensteuer herangezogen, die 2009 eingeführte Abgeltungssteuer entfällt. Selbst genutztes Wohneigentum wird ebenfalls als Einkommen angerechnet, wobei Zins- und Tilgungszahlungen ebenso wie Instandhaltungskosten vorher abgezogen werden (vgl. Mitschke, 2004). ${ }^{6}$ Außerdem werden auch Rentenzahlungen vollständig als Einkommen veranlagt (nach jetzigem Rechtsstand wäre dies erst 2040 der Fall).

\section{Steuertarif}

Unser Vorschlag umfasst eine Abschaffung des Ehengattensplittings sowie die Einführung einer vollständigen Individualbesteuerung, beide Ehepartner werden also mit ihrem individuellen Einkommen veranlagt. ${ }^{7}$ Die Beseitigung des Ehegattensplitting trägt der Erkenntnis von Bach et al. (2011b) Rechnung, wonach eine Begrenzung des Splittingvorteils nicht ausreicht, um die negativen Erwerbsanreize des Ehegattensplittings für Zweitverdiener im Haushalt zu beseitigen. Der Grundfreibetrag in Höhe von 8.004 Euro wird durch eine nicht erstattbare Steuergutschrift in Höhe von 10.000 Euro ersetzt (vgl. Rose, 2011). Der linear-

\footnotetext{
${ }^{5}$ Es ist unklar, ob der Kinderfreibetrag im Zuge einer umfassenden Reform der Lohnnebenkosten weiterhin verfassungsrechtlichen Schutz genießt. Die Berücksichtigung eines Kinderfreibetrags von 7.008 Euro jährlich würde im Vergleich zu unserer Ausgangsvariante jedoch nur zu geringen Aufkommensunterschieden von etwa ein bis zwei Mrd. Euro führen.

${ }^{6}$ Die Einbeziehung selbst genutzten Wohneigentums und Renten halten wir im Hinblick auf eine umfassende Definition der Bemessungsgrundlage für notwendig. Es sei darauf hingewiesen, dass die Aufkommens- und Beschäftigungseffekte unseres Reformvorschlags nicht hierdurch, sondern insbesondere von der Umstellung auf eine Individualbesteuerung (siehe unten) getrieben werden (vgl. Bach et al., 2011b, Eichhorst et al., 2011).

${ }^{7}$ Die Abschaffung der Zusammenveranlagung verheirateter Paare und der Übergang zu einer reinen Individualbesteuerung sind verfassungsrechtlich umstritten. Besteht nach der Scheidung ein Unterhaltsanspruch des einen Ehepartners gegenüber dem anderen, dürfen nach aktueller Rechtsprechung bis zu 13.805 Euro jährlich von der Steuer abgesetzt werden, die der Unterhalt beziehende Partner jedoch versteuern muss. Die Berücksichtigung des so genannten Realsplittings würde im Vergleich zu unserer Ausgangsvariante zu Minderaufkommen von etwa 32 Mrd. Euro führen. Im Vergleich zur derzeitigen Rechtslage würde die Abschaffung des Ehegattensplittings zu steuerlichen Mehreinnahmen von etwa 27 Mrd. Euro führen, das Realsplitting zu rund 5,5 Mrd. Euro (Bach et al., 2011b).
} 
progressive Tarifverlauf der Einkommensteuer wird durch einen Fünf-Stufen-Tarif ersetzt. Ein solcher Tarifverlauf, der bereits mehrfach vorgeschlagen wurde, zeigt in Simulationsstudien in der Regel bessere Arbeitsangebots- und Verteilungswirkungen als Tarife mit weniger Stufen, da aufgrund der höheren Stufenzahl die Entlastungswirkungen dort konzentriert werden können, wo sie positive Beschäftigungseffekte bewirken (vgl. RWI, 2010). In der Ausgangsversion unseres Vorschlags gilt für Einkommen bis 20.000 Euro ein Grenzsteuersatz von 25\%, wobei Einkommen unter 10.000 Euro durch die Steuergutschrift de facto steuerfrei sind. Einkommen zwischen 20.000 Euro und 30.000 Euro werden mit 30\% belastet, von 30.000 Euro bis 40.000 Euro gilt ein Grenzsteuersatz von 40\%, von 40.000 Euro bis 60.000 Euro 50\%. Darüber gilt ein Spitzensteuersatz von 60\%.

In einer modifizierten Fassung unseres Reformvorschlags berücksichtigen wir, dass durch die Verbreiterung der Bemessungsgrundlage in den Sozialversicherungen potentiell neue Ansprüche entstehen könnten, da nun Personen in das System einzahlen, die bisher von der Sozialversicherungspflicht befreit waren (beispielsweise Selbstständige) und somit Leistungsansprüche erwerben, die sie im gegenwärtigen System nicht haben. Die entsprechenden Zusatzkosten lassen sich jedoch ohne detaillierte Informationen über die Alters-, Gesundheits- und Beschäftigungssituation der betreffenden Personengruppen nicht exakt beziffern. Um den potentiellen Mehrbedarf zumindest abzuschätzen, berechnen wir die hypothetischen Arbeitnehmerbeiträge auf die zusätzlichen Einkünfte, die bisher nicht den Sozialversicherungsbeiträgen unterworfen waren. Für alle Versicherungszweige zusammen ergibt sich so eine grobe Schätzung von knapp 40 Mrd. Euro pro Jahr. ${ }^{8}$

\footnotetext{
${ }^{8}$ Dieser Wert ist vergleichbar mit den Berechnungen von Hagist und Raffelhüschen (2003) und Rothgang et al. (2010). Sie geben in ihren Gutachten zur Reform der gesetzlichen Krankenversicherung mögliche Zusatzkosten durch die Einbeziehung vorher Privatversicherter von 16 bis 18 Mrd. Euro an, was unserer Schätzung bezogen auf die Krankenversicherung sehr nahe kommt.
} 
Um das benötigte Mehraufkommen zu generieren, liegen die Grenzsteuersätze in der modifizierten Fassung jeweils fünf Prozentpunkte über den Sätzen im Ursprungsvorschlag (also 30\%, 35\%, 45\%, 55\% und 65\%). Letztlich bleibt aber auch dieser Tarif nur eine sehr grobe Annäherung. Der genaue Tarifverlauf müsste entsprechend auf die tatsächlich entstehenden Kosten abgestimmt werden, um Minderaufkommen $\mathrm{zu}$ vermeiden. Inwieweit überhaupt zusätzliche Ansprüche entstehen oder aber eventuell Leistungen reduziert würden, bliebe zudem eine politische Entscheidung.

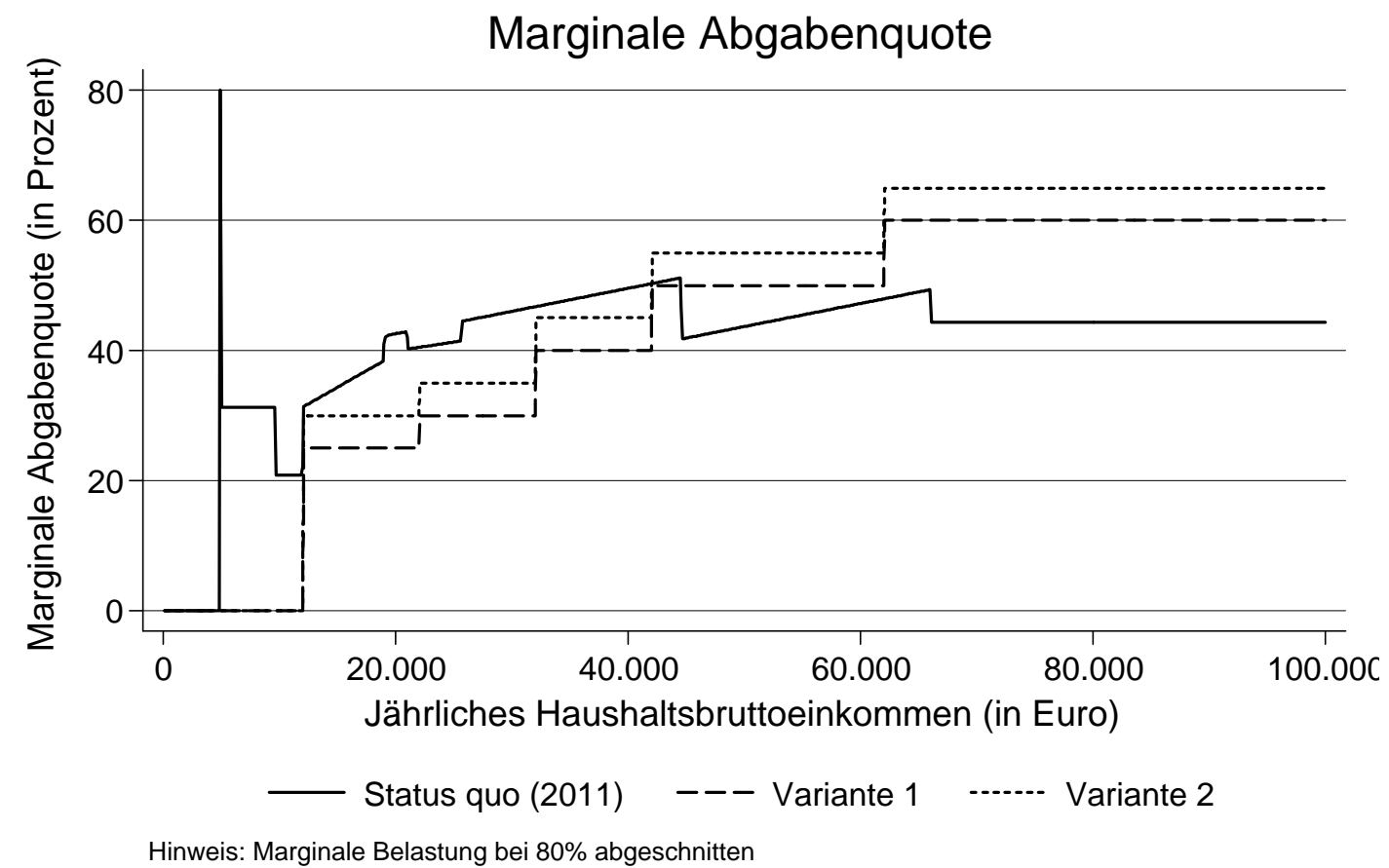

Abbildung 1a: Marginale Abgabensätze in Abhängigkeit des Bruttoeinkommens für einen Single (Quelle: Eigene Darstellung mit IZAYMOD). 


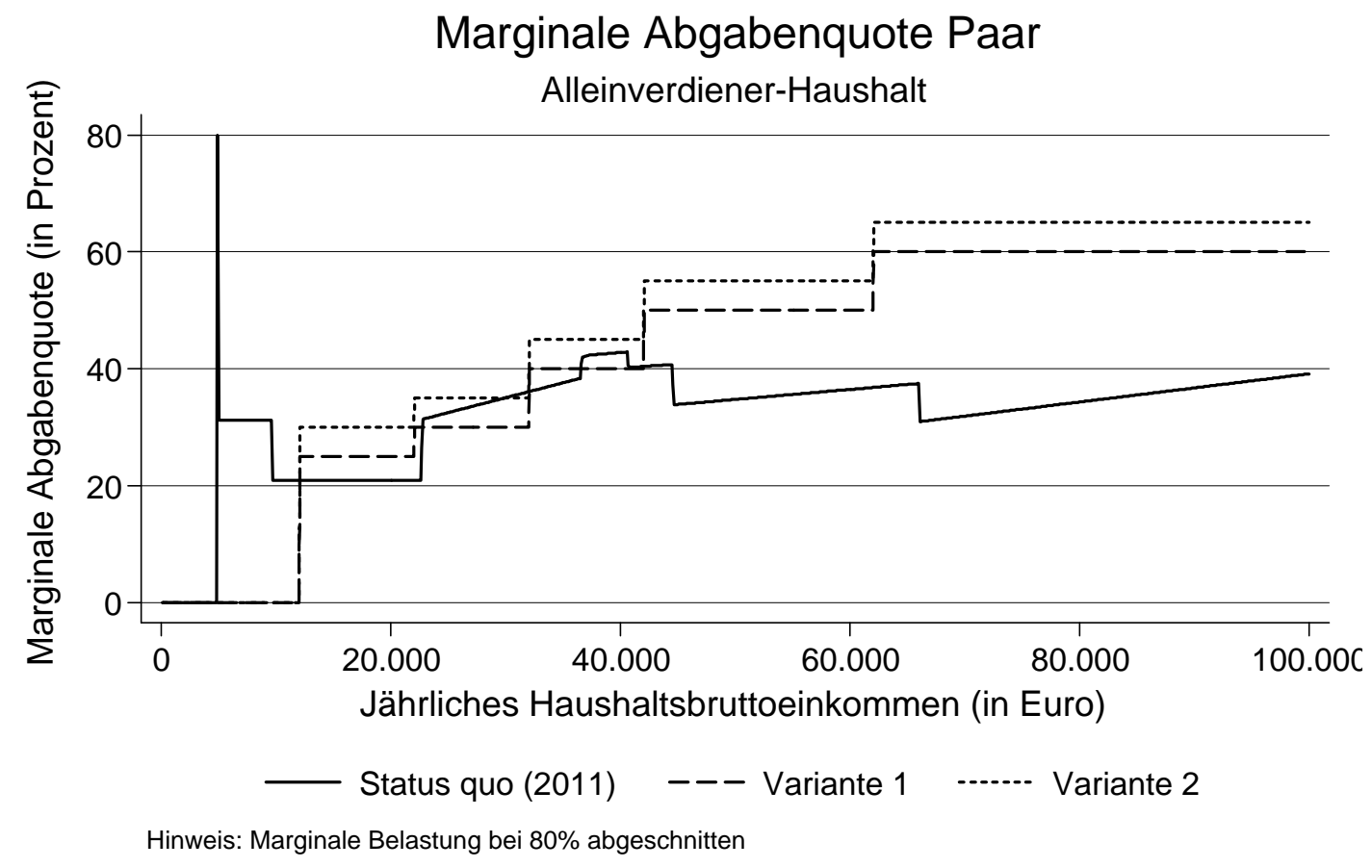

Abbildung 1b: Marginale Abgabensätze des Verdieners in Abhängigkeit seines Bruttoeinkommens für

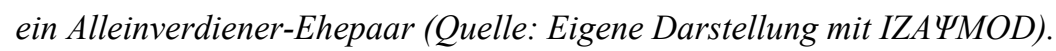

Die Grenzsteuersätze unserer Varianten mögen auf den ersten Blick sehr hoch erscheinen. Hierbei sind jedoch mehrere Punkte zu beachten. Zuerst handelt es sich hierbei jeweils um den Grenz- und nicht den Durchschnittssteuersatz, also um den Steuersatz mit dem ein zusätzlich verdienter Euro belastet wird. Des Weiteren orientieren wir uns an aktuellen Berechnungen für einen (unter fiskalischen Gesichtspunkten) optimalen Spitzensteuersatz (vgl. z.B. Aaberge und Colombino, 2010, Bach et al., 2011a, Landais et al., 2011). Bach et al. (2011a) geben beispielsweise einen optimalen Steuersatz zwischen 58\% und 67\% für Einkommen ab 60.000 Euro an, je nachdem welche Einkunftsarten berücksichtigt werden. Außerdem ist zu beachten, dass die Beiträge zu den Sozialversicherungen ebenfalls enthalten sind, die im Status quo immerhin rund $21 \%$ betragen, und dass der 
Solidaritätszuschlag als Ergänzungsabgabe zur Einkommensteuer entfällt. ${ }^{9}$

Abbildungen 1a und 1b verdeutlichen anhand der marginalen Abgabenquote für einen Single- bzw. einen Paarhaushalt, dass insbesondere für Single-Haushalte die Grenzbelastung trotz der vermeintlich hohen Grenzsteuersätze im unteren Einkommensbereich sogar niedriger ist als im Status quo. Nach aktuellem Rechtsstand entspricht die marginale Abgabenquote dem Grenzsteuersatz inklusive Solidaritätszuschlag plus marginaler Abgabenquote der Sozialversicherungsbeiträge. Bedingt durch die Steuerfinanzierung der Sozialsysteme entspricht die marginale Abgabenquote in den Reformvarianten exakt dem Grenzsteuersatz. Abbildung 1a zeigt deutlich, dass die Grenzbelastung eines Single-Haushalts bis zu einem Jahresbruttoeinkommen von 40.000 Euro in beiden Reformvorschlägen niedriger ist als im Status quo. ${ }^{10}$ Der andere Extremfall eines verheirateten, kinderlosen Paares mit nur einem Verdiener ist in Abbildung 1b dargestellt. Es wird deutlich, dass der Übergang vom Ehegattensplitting zur Individualbesteuerung für diesen Haushaltstyp gerade in mittleren und oberen Einkommensbereichen $\mathrm{zu}$ einer deutlich höheren Grenzbelastung als im Status quo führt. Dies ist vor allem damit zu erklären, dass ein Paarhaushalt mit einem Alleinverdiener im gegenwärtigen System besonders stark vom Splitting profitiert.

Die Kombination aus Steuergutschrift in Höhe von 10.000 Euro und Werbungskostenpauschale von 2.000 Euro führt dazu, dass Erwerbseinkommen bis 12.000 Euro im Jahr (1.000 Euro pro Monat) vollständig abgabenfrei bleiben. Im Vergleich dazu beginnt die Abgabenbelastung im geltenden Rechtsstand bereits bei Einkommen über 400 Euro monatlich und steigt dann stark an. Bei einem

\footnotetext{
${ }^{9}$ Der Zuschlag zur Körperschaftsteuer im Rahmen des Solidaritätszuschlags wird direkt in den Tarif der Körperschaftsteuer integriert.

${ }^{10}$ Die dargestellten Abgabenquoten basieren auf den vereinfachenden Annahmen, dass der Haushalt sein Einkommen ausschließlich aus Erwerbsarbeit erzielt und Beschäftigte nach Überschreiten der Versicherungspflichtgrenze freiwillig in der Gesetzlichen Krankenversicherung verbleiben.
} 
Jahresgehalt von knapp 10.000 Euro wird bereits der volle Beitragssatz zur Sozialversicherung fällig. Zwischen 10.000 und 66.000 Euro verdoppelt sich die prozentuale Belastung aus Steuern und Sozialabgaben, für höhere Einkommen steigt sie nur noch minimal an. Abbildung 2a zeigt die Abgabenquoten der beiden Reformvarianten für kinderlose Singles im Vergleich zum Status quo. Es wird deutlich, dass in der Ursprungsfassung unseres Reformvorschlags Einkommen bis knapp unter 100.000 Euro jährlich (etwa 75.000 Euro in der modifizierten Version) entlastet werden. Lediglich höhere Einkommen werden stärker belastet (siehe auch Abbildung 3). Der Anstieg der Abgabenquote verläuft deutlich langsamer als im Status quo. Im Falle eines verheirateten Alleinverdiener-Paares ohne Kinder hingegen würden Einkommen über 47.000 bzw. 59.000 Euro jährlich stärker belastet als im Status quo (siehe Abbildung 2b).

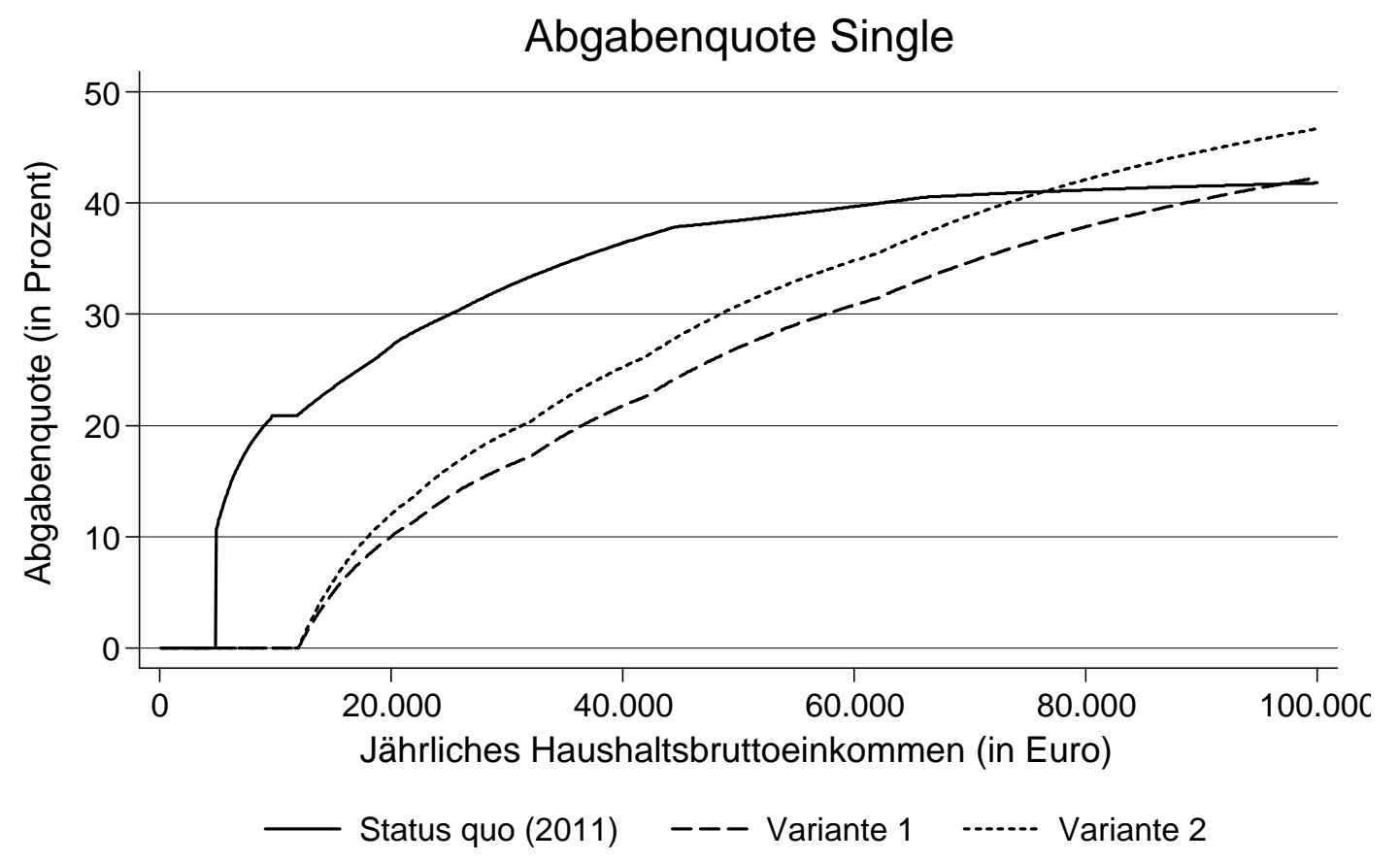

Abbildung 2a: Abgabenquote in Abhängigkeit des Bruttoeinkommens für einen Single (Quelle: Eigene Darstellung mit IZAYMOD) 


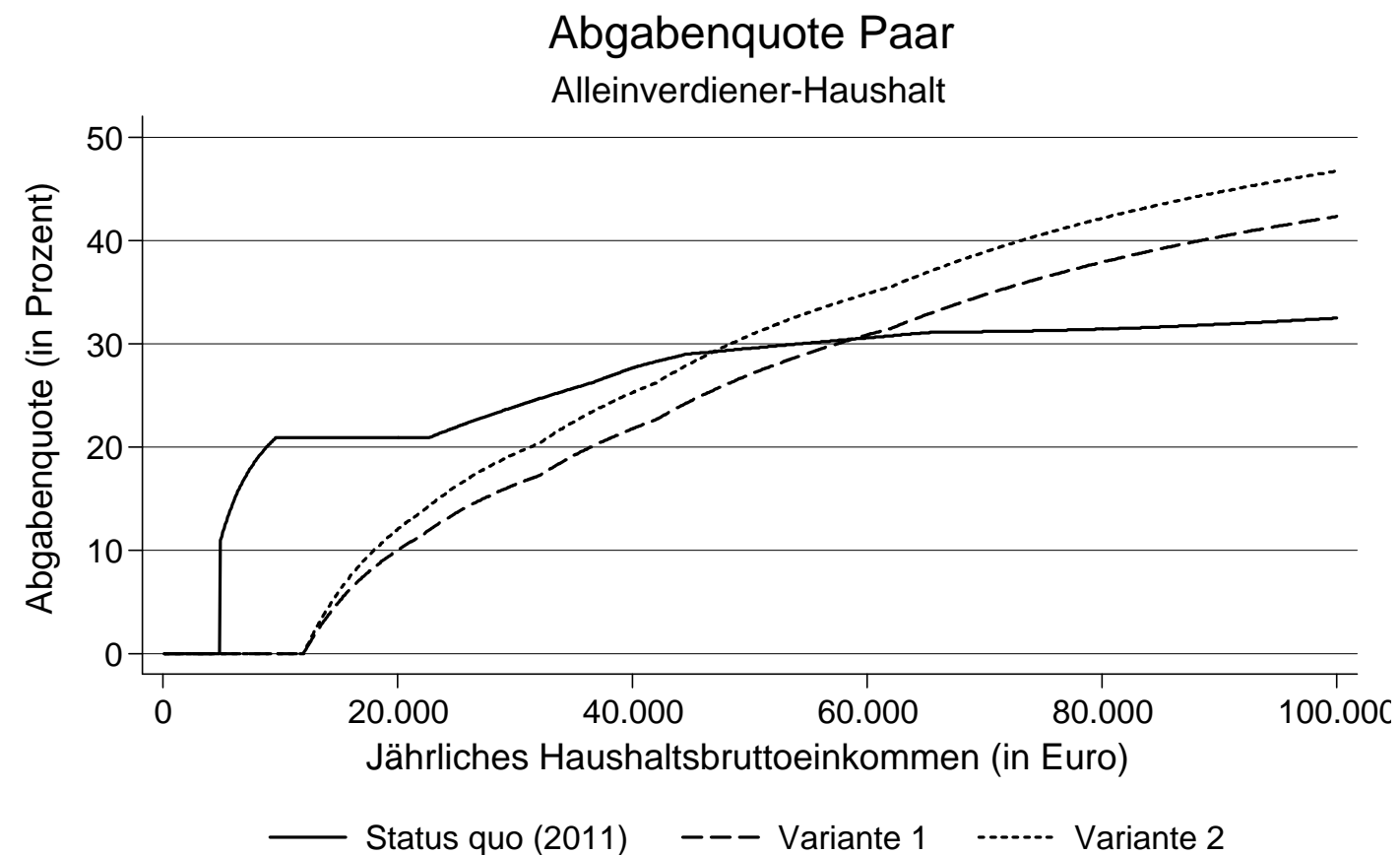

Abbildung 2b: Abgabenquote in Abhängigkeit des Bruttoeinkommens für ein Alleinverdiener-Ehepaar (Quelle: Eigene Darstellung mit IZAYMOD)

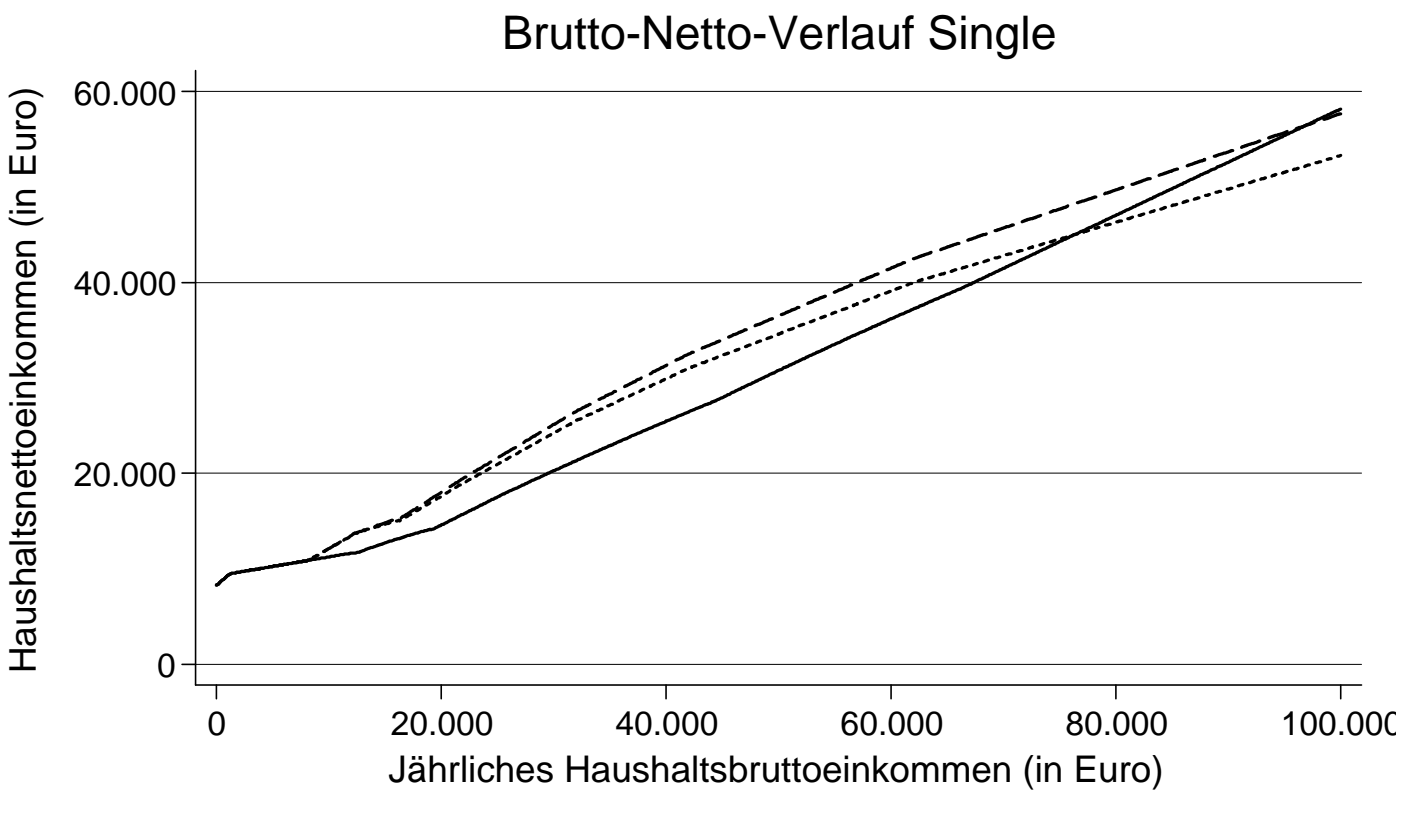

— Status quo (2011) _ _- Variante $1 \quad$-......- Variante 2 
Abbildung 3: Nettoeinkommen in Abhängigkeit des Bruttoeinkommens für einen Single (Quelle: Eigene

Darstellung mit IZAYMOD)

Zusammenfassend lässt sich festhalten, dass sich unser Vorschlag im Vergleich zum Vorschlag von Kirchhof (2011) durch eine noch breitere Bemessungsgrundlage, die Einbeziehung der Sozialabgaben und einen progressiven Tarifverlauf in Form eines Stufen-Tarifs auszeichnet.

\section{Daten und Methodik}

Die vorgestellten Simulationsergebnisse beruhen auf IZAYMOD, dem Mikrosimulationsmodell des IZA. ${ }^{11}$ Dieses erlaubt die Abschätzung der Wirkungen von Veränderungen im Steuer- und Transfersystem auf

(a) das Arbeitsangebot,

(b) die öffentlichen Haushalte (Steueraufkommen, Einnahmen aus Sozialversicherungsbeiträgen und Transferzahlungen) sowie

(c) die Einkommensverteilung.

Mikrosimulationsmodelle gehen über einfache saldenmechanische Abschätzungen deutlich hinaus, da sie Verhaltensanpassungen auf dem Arbeitsmarkt explizit modellieren und somit eine ex ante Abschätzung der Wirkungen zu drei Zeitpunkten erlauben:

1. Sofortwirkungen ohne Verhaltenseffekte (,morning after“-Effekt)

2. Mittelfristige Wirkungen mit Verhaltenseffekt auf das Arbeitsangebot

3. Längerfristige Wirkungen nach Lohn- und Arbeitsnachfrageanpassungen.

\footnotetext{
${ }^{11} \mathrm{Zu}$ Mikrosimulationen vgl. Spahn et al. (1992) und Peichl (2009). Eine ausführliche Dokumentation von IZAYMOD findet sich bei Peichl et al. (2010) und online unter http://www.iza.org/de/webcontent/politics/izapsimod. Das Modell ist in der Lage, das Aufkommen der direkten Steuern und die Kosten der Transferzahlungen relativ genau und detailgetreu abzubilden.
} 
Als Datengrundlage für IZAYMOD dient die 2009er Welle des Sozio-oekonomischen Panels (SOEP, 2009) des Deutschen Instituts für Wirtschaftsforschung (DIW). Die repräsentative Stichprobe der Bevölkerung umfasst über 20.000 Personen in rund 11.000 Haushalten.

In einem ersten Schritt wird das Steuer- und Transfersystem zum Rechtsstand 2011 im Rahmen eines statischen Moduls nachgebildet. Dabei wird unter Berücksichtigung von Freibeträgen, Anrechnungspauschalen, Sonderausgaben sowie Abzugsbeträgen für außergewöhnliche Belastungen und sonstige Privataufwendungen das individuell verfügbare Nettoeinkommen für jeden Fall der Stichprobe unter Berücksichtigung des jeweiligen Haushaltskontextes berechnet. Anschließend werden die Ergebnisse mit den durch die Fortschreibung angepassten Fallgewichten multipliziert und damit auf die Gesamtpopulation hochgerechnet. Genauso werden für die betrachteten Reformvarianten die individuell zu leistenden Einkommensteuerzahlungen und die Nettoeinkommen der Steuerpflichtigen ermittelt. Auf diese Weise können sowohl die Gesamteffekte als auch die Auswirkungen auf jeden einzelnen Steuerfall analysiert werden.

Um die Effekte auf das Arbeitsangebot zu simulieren, verwenden wir ein diskretes Nutzenmodell in Anlehnung an van Soest (1995). Dabei handelt es sich um ein statisches, strukturelles Haushaltsarbeitsangebotsmodell, das die Arbeitsangebotsentscheidung der Haushaltsmitglieder als optimale Wahl zwischen einer begrenzten Anzahl von möglichen Arbeitszeitkategorien modelliert. Konkret können sich die Individuen zwischen Nicht-Erwerbstätigkeit ( 0 Stunden), Teilzeittätigkeit im Umfang von durchschnittlich 10, 20 oder 30 Wochenstunden, 
sowie Vollzeittätigkeit im Umfang von durchschnittlich 40, 50 oder 60 Wochenstunden entscheiden. Paarhaushalte treffen ihre Arbeitsangebotsentscheidung per Annahme gemeinsam auf der Grundlage eines Haushaltsnutzenmodells. Durch Berücksichtigung der möglichen Kombinationen können somit sowohl Wirkungen auf das Arbeitsangebot von Frauen als auch von Männern im Haushaltskontext simuliert werden.

Neben dem benötigten Zeitaufwand hängt der spezifische Nutzen einer Arbeitszeitkategorie vom damit verbundenen Nettoeinkommen ab. Dies wiederum ist abhängig vom individuellen, am Markt erzielbaren Stundenlohn. Dieser lässt sich entweder unmittelbar beobachten oder muss separat vorab geschätzt werden. Es wird unterstellt, dass die jeweils nutzenmaximale Alternative gewählt wird. Unter dieser Prämisse lassen sich die Parameter der Nutzenfunktion empirisch schätzen.

Durch Eingriffe in das Steuer- und Transfersystem verändert sich der Nutzen einzelner Arbeitszeitkategorien, so dass es im Einzelfall zu Verhaltensänderungen kommen kann. Die Arbeitsangebotseffekte ergeben sich als Summe der simulierten nutzenmaximierenden individuellen Entscheidungen vor dem Hintergrund veränderter monetärer Erwerbsanreize.

Die meisten Mikrosimulationsmodelle berücksichtigen nur die mittelfristige Veränderung des Arbeitsangebotes auf Grund des veränderten Nettoeinkommens und abstrahieren von Arbeitsnachfrageeffekten. Eine solche Vereinfachung ist allerdings nur unter der restriktiven Annahme einer perfekt elastischen Arbeitsnachfrage zulässig. Da allerdings aus zahlreichen empirischen Studien hervorgeht, dass die 
Arbeitsnachfragekurve keineswegs perfekt elastisch, sondern fallend verläuft ${ }^{12}$, unterscheidet sich der letztendliche Beschäftigungseffekt vom Arbeitsangebotseffekt.

Aus diesem Grund verfügt IZAYMOD über ein eigenes Arbeitsnachfragemodul, das es ermöglicht, die über die mittlere Frist hinausgehenden Wirkungen nach Anpassung der Arbeitsnachfrage und des Lohnes zu simulieren (vgl. Peichl und Siegloch, 2012). Hierzu werden auf Basis der Linked Employer-Employee-Daten (LIAB) des Instituts für Arbeitsmarkt- und Berufsforschung Arbeitsnachfragefunktionen für verschiedene Qualifikationstypen und Sektoren geschätzt. Diese werden dann mit dem Arbeitsangebotsmodell verknüpft. Unter der Annahme eines flexiblen Arbeitsmarktes induzieren Änderungen des Arbeitsangebotes Lohnanpassungen auf der Arbeitsnachfrageseite. Es wird unterstellt, dass das Gleichgewicht erreicht ist, wenn die ursprüngliche Differenz zwischen Arbeitsangebot und Arbeitsnachfrage wieder hergestellt wird. Die entsprechenden Lohnänderungen wirken anschließend wieder auf das Arbeitsangebot zurück und vice versa. Dieser Prozess wird so lange iteriert, bis die Veränderungen ein vorgegebenes Konvergenzkriterium unterschreiten. Die Wirkungen nach Arbeitsnachfrageanpassungen entsprechen somit dem langfristigen Beschäftigungseffekt, der sich einstellt, wenn Angebot und Nachfrage über Lohnanpassung zum Ausgleich gebracht werden und sich der Arbeitsmarkt wieder im partiellen Gleichgewicht befindet.

Die Schätzungen und Simulationen des Arbeitsangebots- und Arbeitsnachfragemodells beschränken sich auf die erwerbsfähige Bevölkerung, da nur diese dem Arbeitsmarkt zur Verfügung steht. Die Budget- und Verteilungswirkungen

\footnotetext{
${ }^{12}$ Vgl. u.a. Addison et al. (2009), Freier und Steiner (2010) und Peichl und Siegloch (2012).
} 
werden für die Gesamtbevölkerung simuliert und enthalten somit auch die fiskalischen Effekte der Anhebung des Rentenbesteuerungsanteils.

Das zugrunde liegende Simulationsmodell ist in der Lage, die Steuer- und Transferzahlungen des geltenden Rechts relativ exakt (sowohl auf der Mikro- als auch der Makroebene) abzubilden. ${ }^{13}$ Die gewählte Methodik erlaubt es jedoch nicht, die möglichen Auswirkungen auf die Bruttoeinkünfte, die über verändertes Arbeitsangebotsverhalten hinausgehen, zu simulieren. Dazu zählen beispielsweise Verhaltensänderungen im Hinblick auf die (ggf. verminderte) Hinterziehung bzw. Vermeidung von Steuern oder auch eine Verlagerung zwischen Einkunftsarten (,income shiftig“, vgl. z.B. Feenberg und Poterba, 1993, oder Slemrod, 1996). Trotz dieser methodischen Einschränkung lassen sich dennoch Größenordnungen für die Effekte auf das Steueraufkommen sowie auf die Einkommensverteilung abschätzen, die Schlussfolgerungen im Hinblick auf die politische Akzeptanz erlauben.

\section{Simulationsergebnisse}

In diesem Abschnitt werden die Simulationsergebnisse für unseren Reformvorschlag sowie die modifizierte Variante präsentiert. In Tabelle 1 sind für beide Varianten die Auswirkungen auf die öffentlichen Haushalte dargestellt. Dabei differenzieren wir zwischen dem statischen Effekt (vor Verhaltensanpassung) sowie den Wirkungen nach Anpassung des Arbeitsangebots und schließlich der Arbeitsnachfrage. Die Simulation der Grundfassung unseres Vorschlags (Variante 1) führt zu einem leichten Mehraufkommen von etwa vier bis sechs Mrd. Euro. Es wird deutlich, dass die

\footnotetext{
${ }^{13}$ Zum einen liegen die Abweichungen auf der Makroebene im Vergleich der aggregierten Steuer- und Transferzahlungen zu den vom Bundesminesterium der Finanzen ausgewiesenen Werten in der Regel bei unter $1 \%$ ohne dass es erkennbar zu systematischen Über- oder Unterschätzungen kommt. Auch auf der Mikroebene, also dem Vergleich der simulierten mit den in den SOEP-Daten ausgewiesenen Steuer- und Transferzahlungen liegen die maximalen Abweichungen bei unter 5\%, im Durchschnitt bei rund $1 \%$.
} 
Verbreiterung der Bemessungsgrundlage und der neue Tarifverlauf die Mindereinnahmen durch die Abschaffung der Sozialversicherungen deutlich ausgleichen. Das Minus bei den Sozialversicherungen besteht lediglich im Wegfall der Arbeitnehmerbeiträge, da das Aufkommen aus den Arbeitgeberbeiträgen wie oben beschrieben über eine veränderte Unternehmensbesteuerung ausgeglichen wird und so keine Auswirkungen auf unsere Budgeteffekte hat. Tabelle 1 zeigt zudem, dass die Ausgaben für Arbeitslosengeld II sinken (zwischen drei und fünf Mrd. Euro). Dieses Ergebnis ist auf die deutliche Absenkung der Steuer- und Abgabenbelastung für niedrige Einkommen zurückzuführen. Diese entlastet nicht zuletzt erwerbstätige Transferempfänger vor allem von den SV-Beiträgen. Damit verbleibt ihnen ein größerer Teil selbst erworbenen Einkommens, so dass der Staat weniger zur Erreichung des Existenzminimums (also des Regelbedarfs) aufstocken muss. ${ }^{14}$ Verstärkt wird dies noch durch den positiven Beschäftigungseffekt unseres Reformvorschlags (siehe Tabelle 2). Variante 2 unseres Reformvorschlags führt wie bereits oben diskutiert zu einem Mehraufkommen von rund 50 Mrd. Euro.

Tabelle 1: Budgeteffekte (in Mrd. Euro)

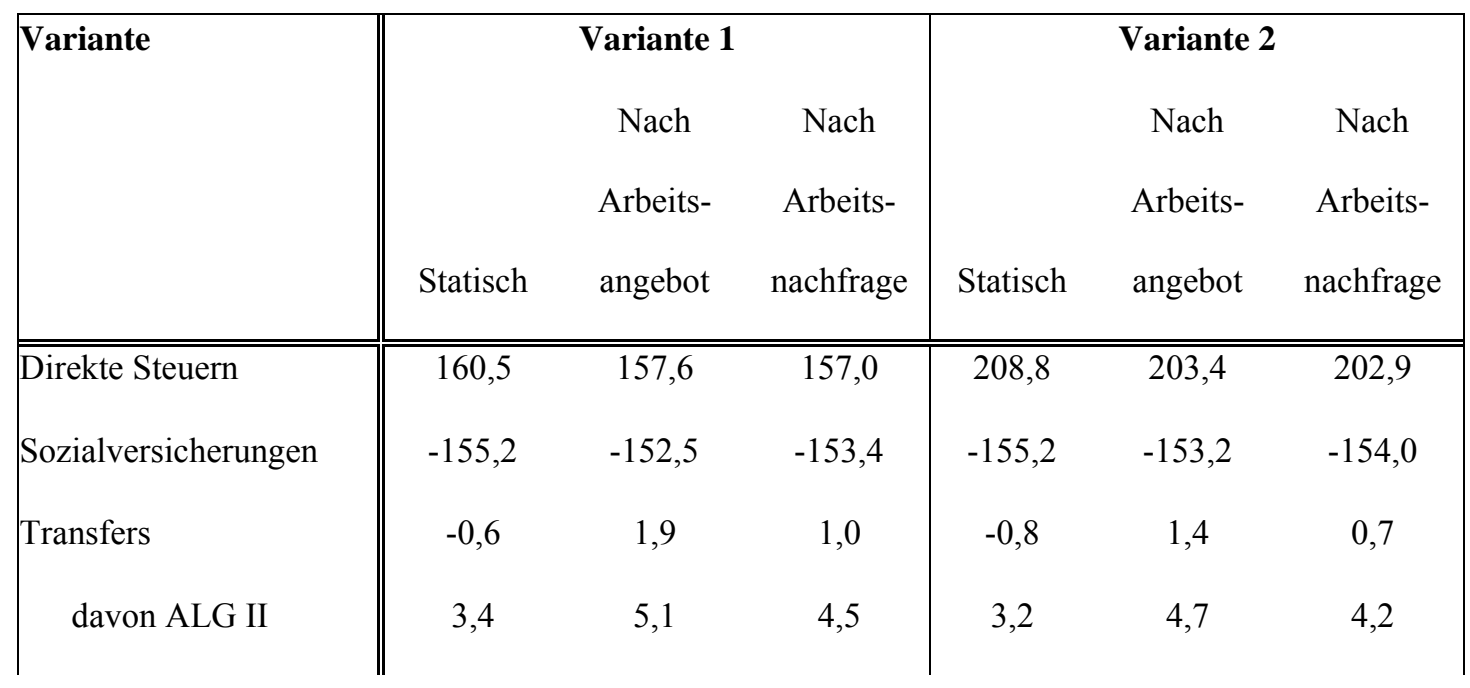

\footnotetext{
${ }^{14}$ Der annähernd neutrale Gesamteffekt bei den Transferzahlungen kommt durch den Wegfall des Kinderfreibetrages und dem damit verbundenen Anstieg der Kindergeldzahlungen zustande.
} 


\begin{tabular}{|l||ccc|ccc|} 
Gesamt & $\mathbf{4 , 1}$ & $\mathbf{6 , 5}$ & $\mathbf{4 , 1}$ & $\mathbf{5 2 , 1}$ & $\mathbf{5 1 , 0}$ & $\mathbf{4 9 , 0}$ \\
\hline
\end{tabular}

In Variante 1 steigt das Arbeitsangebot der Haushalte um über 830.000 Vollzeitäquivalente, in der Alternativvariante immer noch um 672.000. Die Differenz lässt sich vor allem auf die höheren Grenzsteuersätze der modifizierten Variante zurückführen. Die Auswirkungen auf die Partizipation am Arbeitsmarkt sind ebenfalls durchweg positiv: In beiden Varianten steigt die Zahl der am Arbeitsmarkt teilnehmenden Personen um über 750.000, die Partizipationsrate um etwa zwei Prozentpunkte. Im Detail zeigt sich, dass unser Reformvorschlag für all jene Bevölkerungsgruppen starke Anreize schafft, das Arbeitsangebot auszuweiten, die bisher gar nicht oder nur in geringem Ausmaß arbeiten. Dieser Effekt überkompensiert auch die negativen Erwerbsanreize, die durch die Anhebung des Spitzensteuersatzes für Beschäftigte mit sehr hohen Einkommen entstehen. ${ }^{15}$

Tabelle 2: Arbeitsangebots- und Beschäftigungseffekte

\begin{tabular}{|l||cc|cc|}
\hline Variante & \multicolumn{2}{|c|}{ Variante 1 } & \multicolumn{2}{c|}{ Variante 2 } \\
& Arbeitsangebot & Beschäftigung & Arbeitsangebot & Beschäftigung \\
\hline \hline Vollzeitäquivalente (in Tsd.) & 837,4 & 525,5 & 672,0 & 422,9 \\
Partizipation (in Tsd.) & 771,2 & 497,0 & 755,6 & 537,6 \\
Partizipationsrate (in \%-Punkten) & 2,1 & 1,3 & 2,0 & 1,4 \\
\hline
\end{tabular}

\footnotetext{
${ }^{15}$ Die empirische Literatur zur Messung von Arbeitsangebotselastizitäten zeigt, dass Bezieher niedriger Einkommen bei gleicher Einkommensänderung stärker reagieren als Besserverdiener (siehe z.B. Aaberge und Colombino, 2010, und Bargain et al., 2011).
} 
Tabelle 2 zeigt zudem, dass die Anpassung der Arbeitsnachfrage den positiven Beschäftigungseffekt um etwa 35\% reduziert. Ein solcher Effekt ist zu erwarten, da ein derart starker Arbeitsangebotseffekt unausweichlich zu Anpassungen des Lohnniveaus führt (vgl. Peichl und Siegloch, 2012). Die Lohnanpassungen betreffen in erster Linie niedrige und mittlere Einkommen, die auf Grund der starken Entlastungen ihr Arbeitsangebot in Relation stärker ausweiten. Die Reaktion der Arbeitsnachfrage führt ebenfalls zu einer geringfügigen Reduktion der positiven Budgetwirkung (siehe Tabelle 1).

Im Folgenden untersuchen wir die Auswirkungen der beiden vorgeschlagenen Reformvarianten auf die personelle Einkommensverteilung. ${ }^{16}$ Tabelle 3 zeigt anhand der Position in der Einkommensverteilung die Gewinner und Verlierer der Reformvarianten. Dabei wird das jeweilige Durchschnittseinkommen (für die Reformen nach Arbeitsangebotsanpassung) auf 100\% normiert und die Durchschnittseinkommen der einzelnen Dezile in Relation dazu gesetzt. Es zeigt sich, dass im Status quo die oberen vierzig Prozent der Bevölkerung überdurchschnittliche und die unteren sechzig Prozent unterdurchschnittliche Einkommen aufweisen. So verfügt allein das oberste Zehntel der Einkommensverteilung im Mittel über ein Einkommen, das fast dem 2,5-fachen Durchschnittseinkommen entspricht. Für unseren Reformvorschlag sowie die modifizierte Variante zeigt sich, dass lediglich das oberste Dezil stärker belastet wird. Deren Einkommen würde im Vergleich zum mittleren Einkommen um 27,1 bzw. 33,8 Prozentpunkte sinken, wäre aber immer noch mehr als doppelt so hoch wie das Durchschnittseinkommen. Im Gegensatz dazu würden die übrigen $90 \%$ der Einkommensverteilung von der vorgeschlagenen Reform

\footnotetext{
${ }^{16}$ Die Verteilungseffekte basieren auf äquivalenzgewichteten Nettoeinkommen (modifizierte OECDÄquivalenzskala).
} 
profitieren. Ihre Einkommen würden im Verhältnis zum Durchschnitt steigen, insbesondere gilt dies für die mittleren Dezile (die Mittelklasse) mit einem Anstieg zwischen vier und sechs Prozentpunkten.

Tabelle 3: Verteilungseffekte nach Beschäftigungsanpassung I

\begin{tabular}{|l||ccc|cc|}
\hline \multicolumn{1}{|||}{} & \multicolumn{3}{c|}{ Relation zum Durchschnitt } & \multicolumn{2}{c|}{ Veränderung } \\
& Status quo & Variante 1 & Variante 2 & Variante 1 & Variante 2 \\
\hline \hline Gesamt & 100,0 & 100,0 & 100,0 & & \\
Ärmste 10\% & 40,2 & 41,5 & 43,1 & 1,3 & 2,8 \\
Dezil 2 & 51,1 & 52,5 & 54,2 & 1,4 & 3,1 \\
Dezil 3 & 60,2 & 62,7 & 64,1 & 2,5 & 3,8 \\
Dezil 4 & 70,2 & 72,9 & 73,9 & 2,7 & 3,7 \\
Dezil 5 & 79,5 & 82,8 & 83,7 & 3,3 & 4,3 \\
Dezil 6 & 91,0 & 95,1 & 95,8 & 4,1 & 4,9 \\
Dezil 7 & 104,4 & 110,2 & 110,5 & 5,8 & 6,1 \\
Dezil 8 & 120,8 & 125,0 & 124,9 & 4,2 & 4,1 \\
Dezil 9 & 145,1 & 146,7 & 145,5 & 1,6 & 0,4 \\
Reichste 10\% & 246,8 & 219,7 & 213,0 & $-27,1$ & $-33,8$ \\
\hline
\end{tabular}

Quelle: Eigene Berechnungen mit IZAYMOD

Die Besserstellung der Mittelschicht zeigt sich auch anhand der Kerndichteschätzung der äquivalenzgewichteten monatlichen Nettoeinkommen, dargestellt in Abbildung 4. Die beiden Reformen führen dazu, dass die Dichte der Haushalte mit einem Nettoeinkommen zwischen 2.000 und 3.500 Euro im Vergleich zum Status quo erkennbar ansteigt. Im Gegensatz dazu gibt es nach der Reform insbesondere in Variante 2 weniger Haushalte mit einem Nettoeinkommen von mehr als 4.000 Euro, 
während in Variante 1 die Dichte der Haushalte im Einkommensbereich zwischen ca. 750 Euro und ca. 1.500 Euro deutlich zurück geht

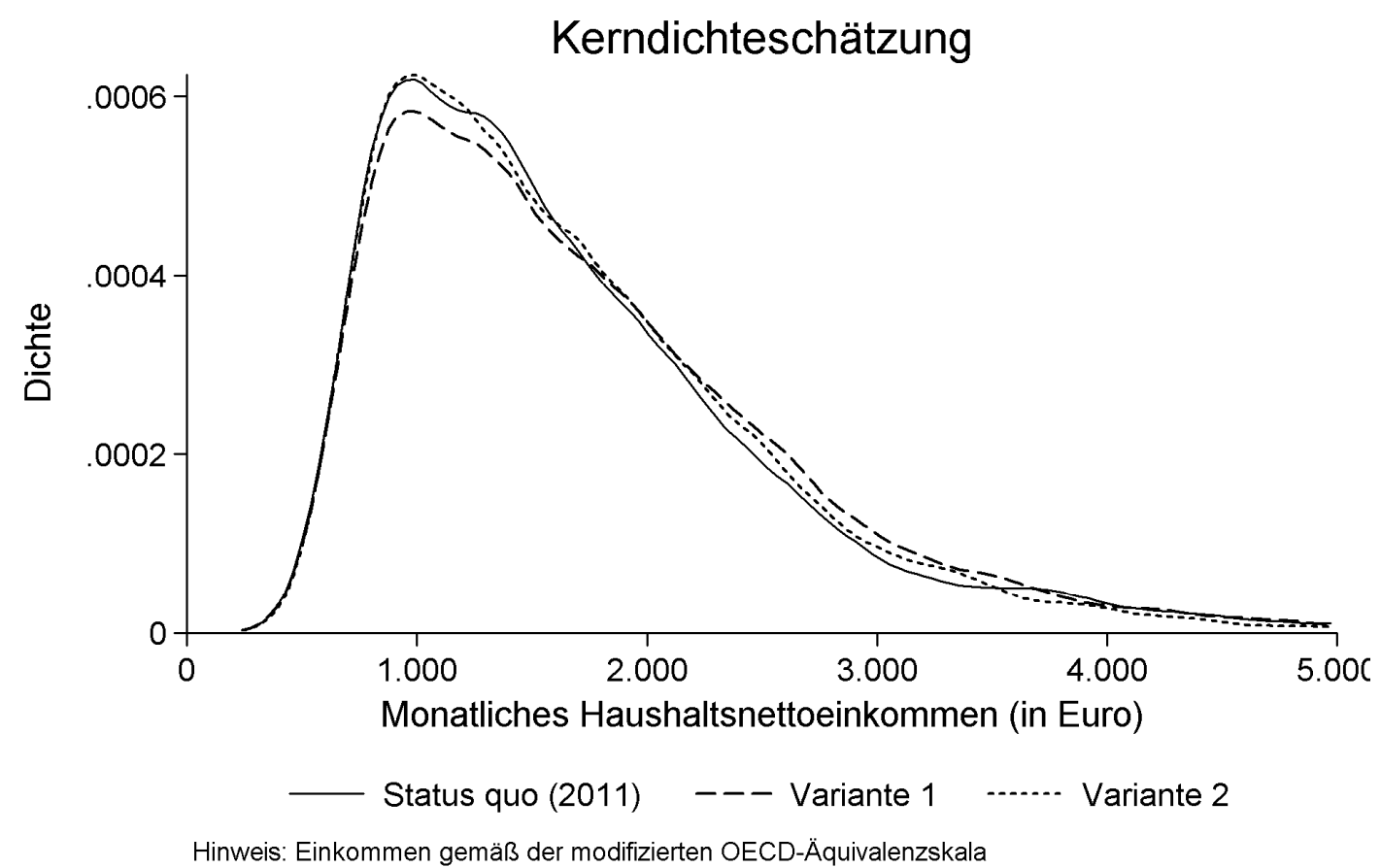

Abbildung 4: Kerndichteschätzung des äquivalenzgewichteten Haushaltsnettoeinkommens (Quelle: Eigene Darstellung mit IZAYMOD)

Diese Veränderungen lassen bereits den Schluss $\mathrm{zu}$, dass der vorgestellte Reformvorschlag zu einer Reduktion der Einkommensungleichheit führt. Tabelle 4 gibt einen Überblick über wichtige Verteilungsmaße wie den Gini-Koeffizienten und die Perzentil-Verhältnisse. In der Ursprungsvariante fällt der Gini-Index von 0,30 (Status quo) auf 0,285, bzw. 0,275 in angepassten Fassung. Mit Blick auf das Verhältnis der Perzentile fällt das Ergebnis etwas gemischter aus. In der Ursprungsfassung steigt das Verhältnis P90/P10 leicht, während es in der modifizierten Variante sinkt. Betrachtet man jedoch beide Hälften der Einkommensverteilung getrennt voneinander, fällt auf, dass die Ungleichheit in der 
oberen Einkommenshälfte in beiden Varianten sinkt (-0,031 bzw. -0,076), während sie in der unteren Hälfte leicht steigt bzw. nur marginal sinkt (0,051 bzw. -0,001).

Tabelle 4: Verteilungseffekte nach Beschäftigungsanpassung II

\begin{tabular}{|l||ccc|cc|}
\hline \multicolumn{1}{|c||}{} & \multicolumn{3}{c|}{ Einkommensungleichheit } & \multicolumn{2}{c|}{ Veränderung } \\
& Status quo & Variante 1 & Variante 2 & Variante 1 & Variante 2 \\
\hline \hline Gini & 0,300 & 0,285 & 0,275 & $-0,015$ & $-0,025$ \\
P90/P10 & 3,531 & 3,570 & 3,391 & 0,040 & $-0,140$ \\
P90/P50 & 1,938 & 1,907 & 1,862 & $-0,031$ & $-0,076$ \\
P50/P10 & 1,822 & 1,872 & 1,821 & 0,051 & $-0,001$ \\
\hline
\end{tabular}

Quelle: Eigene Berechnungen mit IZAYMOD

Die Verteilungswirkungen begünstigen also vornehmlich die Mittelschicht. Dies führt zu einem Absinken der Ungleichheit im Vergleich zu den Spitzeneinkommen, die zusätzlich noch stärker besteuert werden (P90/P50-Verhältnis). Gleichzeitig gewinnt die Mittelschicht aber auch gegenüber den ärmsten zehn Prozent, deren Einkommen sich verhältnismäßig wenig verändert (s. auch Abbildung 4). Um auch in den unteren Dezilen einen größeren Anstieg des Durchschnittseinkommens zu erreichen, müsste bei den Transfersystemen angesetzt werden, statt nur das Steuer- und Abgabensystem in den Blick zu nehmen.

\section{Fazit}

Das deutsche Einkommenssteuerrecht ist zu komplex und zu intransparent. Zahlreiche Reformkonzepte wurden in den vergangenen Jahren öffentlich diskutiert. Allerdings ist es bisher keinem gelungen, den bestehenden Zielkonflikt $\mathrm{zu}$ überwinden und positive Beschäftigungs- und Verteilungswirkungen bei einem neutralen Effekt auf 
den Staatshaushalt zu erzielen. Ein Kernproblem der bisher gemachten Vorschläge ist, dass sie die Sozialversicherungsabgaben und deren Interaktion mit der Einkommensteuer vernachlässigen. Dadurch wurde ein großer Teil der Abgaben an den Staat bisher nicht berücksichtigt.

Unser Reformvorschlag setzt genau an dieser Stelle an und schlägt ein integriertes Steuer- und Abgabensystem vor, das eine Steuerfinanzierung der Sozialsysteme beinhaltet. Gleichzeitig führen wir die Individualbesteuerung ein, ersetzen den komplizierten linear-progressiven Einkommensteuertarif durch einen weitaus einfacheren Stufentarif und schaffen praktisch alle Abzugsmöglichkeiten bei der Bestimmung des zu versteuernden Einkommens ab. Es zeigt sich, dass durch diese umfassende Reform der erwähnte Zielkonflikt überwunden werden kann.

Erstens hat die Reform positive Beschäftigungswirkungen in einem Ausmaß von 420.000 bis 520.000 Vollzeitäquivalenten. ${ }^{17}$ Dieser Effekt entspricht fast vollständig dem damit verbundenen Partizipationseffekt, d.h. das zusätzliche Arbeitsvolumen entsteht nicht in erster Linie durch eine Ausweitung der pro Kopf gearbeiteten Stunden, sondern durch die Schaffung von zusätzlichen Beschäftigungsmöglichkeiten für insgesamt etwa 500.000 Personen. Dieses Ergebnis ist insofern bemerkenswert, als dass viele in der Vergangenheit gemachten Reformkonzepte eine Spreizung auf dem Arbeitsmarkt nach sich ziehen würden: Entweder steigt die Anzahl der gesamten Arbeitsstunden oder die Teilnahme am Arbeitsmarkt. Unser Vorschlag erhöht sowohl das Arbeitsvolumen als auch die Partizipation.

\footnotetext{
${ }^{17}$ Dabei wird angenommen, dass eine Vollzeit arbeitende Person 40 Stunden pro Woche arbeitet.
} 
Zweitens würde die Ungleichheit durch höhere Grenzsteuersätze im oberen Bereich der Einkommensverteilung sinken. Insbesondere die Mittelschicht, die im Status Quo überproportional durch Sozialabgaben und Steuerzahlungen belastet wird, würde ihre relative Nettoeinkommensposition verbessern. Der Reformvorschlag verschlankt somit den so genannten Mittelstandsbauch und erhöht stattdessen die Belastung der obersten zehn Prozent der Einkommensverteilung. Dies entspricht aktuellen Forderungen in der nationalen und internationalen Steuerdebatte, die reichsten Bevölkerungsgruppen stärker bei der Finanzierung der Folgen der Finanzkrise heranzuziehen. Es sei jedoch an dieser Stelle darauf hingewiesen, dass es möglich ist, ein aufkommensneutrales System zu entwickeln, dass höhere Einkommen mit einem Grenzsteuersatz von beispielsweise maximal 50\% belastet. Auch ein solches System könnte, je nach Ausgestaltung, die Ungleichheit senken und die Beschäftigung erhöhen. Dieser Beitrag soll vor allem aufzeigen, welche Spielräume ein integriertes Steuer- und Abgabensystem unter Berücksichtigung theoretisch optimaler Grenzsteuersätze schaffen könnte. Die letztliche Wahl der exakten Tariffunktion wird immer eine politische Entscheidung sein.

Drittens würde der Vorschlag nicht zu einer zusätzlichen Belastung des Staatshaushaltes führen. Beide Reformvarianten verzeichnen sogar ein leichtes Plus bei den Staatseinnahmen. Dieses Mehraufkommen könnte beispielsweise zum Ausbau von Kinderbetreuungsangeboten genutzt werden. Dies wäre insbesondere im Hinblick auf die stark positiven Arbeitsangebotsanreize für Mütter notwendig. Darüber hinaus sollten Mehreinnahmen zur Reduzierung der Nettoneuverschuldung verwendet werden und so einen Beitrag zur Einhaltung der Schuldenbremse leisten. Mittelfristig wären noch höhere Steuereinnahmen zu erwarten, da Doppelstrukturen abgebaut und 
somit Administrationskosten eingespart werden können. Das hier vorgeschlagene integrierte Steuer- und Abgabensystem reduziert den Aufwand bei der Steuererklärung erheblich („Erklärung auf dem Bierdeckel“). Darüber hinaus würde der Abbau der Komplexität des Steuer- und Abgabensystems zu weniger Schlupflöchern und somit zu weniger Steuervermeidung führen.

Die mittelfristigen Mehreinnahmen sollten unbedingt zur Tilgung der langfristigen Staatsschulden verwendet und nicht in Steuererleichterungen umgemünzt werden. Die aktuelle fiskalische Situation, die europäische Schuldenkrise und die seit 2009 im Grundgesetz verankerte Schuldenbremse haben den Spielraum für Steuererleichterungen ohnehin stark eingeschränkt. Die Vorstellung, dass Steuersenkungen selbstfinanzierend sind, weil sie $\mathrm{zu}$ mehr Wirtschaftswachstum führen, konnte empirisch bisher nicht belegt werden (vgl. auch Fuest, 2011). Steuersenkungen entfalten zwar durchaus positive Konjunkturwirkungen, diese sind aber insbesondere bei der Einkommensteuer zu gering, um eine Selbstfinanzierung zu ermöglichen.

Außerdem ist die Versuchung groß, durch Steuergeschenke entsprechende Klientelpolitik zu betreiben. Ein einfacheres Steuerrecht würde diese Möglichkeiten erheblich einschränken, da in einem solchen System die Verteilungswirkungen von Steuerentlastungen besser nachvollziehbar sind. Höhere Transparenz macht Klientelpolitik leichter identifizierbar und dürfte zu größeren Widerständen in der Bevölkerung führen als in einem sehr instransparenten System, in dem nur schwer nachvollziehbar ist, von wem zu wem umverteilt wird. Dies ist auch deshalb wichtig, weil bei hoher Staatsverschuldung und einer alternden Bevölkerung jedes 
Steuergeschenk immer einen großen Verlierer hervorbringen wird - die zukünftigen Generationen, die für die Schulden letztlich aufkommen müssen, sich aber im politischen Prozess jetzt noch nicht artikulieren können. Dies kann durch ein einfacheres Steuerrecht verhindert werden. 


\section{Literatur}

Aaberge, R., U. Colombino (2010): Using a Microeconometric Model of Household Labour Supply to Design Optimal Income Taxes, Carlo Alberto Notebooks 157, Collegio Carlo Alberto.

Addison, J. T., L. Bellmann, T. Schank, P. Teixeira (2009): The Demand for Labor: An Analysis Using Matched Employer-Employee Data from the German LIAB. Will the High Unskilled Worker Own-Wage Elasticity Please Stand Up?, Journal of Labor Research 29(2), S. 114-137.

Arnold, J., B. Brys, C. Heady, A. Johansson, C. Schwellnus, L. Vartia (2011): Tax Policy for Economic Recovery and Growth. The Economic Journal, 121(550), S. F59-F80.

aus dem Moore, N., R. Kambeck, T. Kasten (2009): Auswirkungen der Steuerprogramme zur Bundestagswahl 2009 - Eine mikrodatenbasierte Analyse der Reformvorschläge von CDU/CSU, SPD und FDP. RWI Materialien, Heft 55.

aus dem Moore, N., B. Beimann, P. David, R. Kambeck, T. Kasten (2010): Evaluation des Vorschlags der FDP vom 13. April 2010 für einen Stufentarif der Einkommensteuer, Projektbericht Rheinisch-Westfälisches Institut für Wirtschaftsforschung.

Bach, S. (2005): Grundlegende Reform der Einkommensbesteuerung: Inwieweit kann die Bemessungsgrundlage verbreitert und das Steuerrecht vereinfacht werden?, DIW Wochenbericht Nr. 36/2005.

Bach, S., V. Steiner, D. Teichmann (2002): Berechnungen zum Reformvorschlag „Arbeit für viele“, Gutachten des DIW Berlin im Auftrag des Nachrichtenmagazins DER SPIEGEL.

Bach, S., P. Haan, H.-J. Rudolph, V. Steiner (2004): Reformkonzepte zur Einkommens- und Ertragsbesteuerung: Erhebliche Aufkommens- und Verteilungswirkungen, aber relativ geringe Effekte auf das Arbeitsangebot, DIW Wochenbericht Nr.16/2004. 
Bach, S., G. Corneo, V. Steiner (2011a): Optimal top marginal tax rates under income splitting for couples. CEPR Discussion Paper No. 8435, London, Centre for Economic Policy Research.

Bach, S., J. Geyer, P. Haan, K. Wrohlich (2011b): Reform des Ehegattensplittings: Nur eine reine Individualbesteuerung erhöht die Erwerbsanreize deutlich, DIW Wochenbericht Nr. 41/2011.

Bargain, O., K. Orsini, A. Peichl (2011): Labor Supply Elasticities in Europe and the US, IZA Discussion Paper No. 5820.

Eichhorst, W., M. J. Kendzia, A. Peichl, N. Pestel, S. Siegloch, V. Tobsch (2011): Aktivierung von Fachkräftepotenzialen: Frauen und Mütter, Kurzexpertise im Auftrag des Bundesministeriums für Arbeit und Soziales, IZA Research Report Nr. 39.

FDP (2008): Die gerechte Steuer: Einfach, niedrig und sozial. Das Nettokonzept der FDP, Beschluss vom 59. Ordentlichen Bundesparteitag.

FDP (2009): Die Mitte stärken. - Deutschlandprogramm der Freien Demokratischen Partei.

Feenberg, D. and J. Poterba (1993): Income inequality and the incomes of very high income taxpayers: Evidence from tax returns, in Tax Policy and the Economy 7, edited by James M. Poterba, M.I.T. Press, S. 145-177.

Fuest, C. (2011): Einkommensteuersenkungen: Rückfall in Fehler der Vergangenheit, Wirtschaftsdienst 91(7), S. 434-435.

Fuest, C., A. Peichl, T. Schaefer (2007a): Die Flat Tax: Wer gewinnt? Wer verliert? Eine empirische Analyse für Deutschland, Steuer und Wirtschaft, 1/2007, S. 22-29.

Fuest, C., A. Peichl, T. Schaefer (2007b): Führt Steuervereinfachung zu einer „gerechteren“ Einkommensverteilung? Eine empirische Analyse für Deutschland, Perspektiven der Wirtschaftspolitik 8(1), S. 20-37.

Fuest, C., A. Peichl, T. Schaefer (2008b): Does Tax Simplification yield more Equity and Efficiency? An empirical analysis for Germany, CESifo Economic Studies 54(1), S. 73-97.

Freier, R., V. Steiner: "Marginal employment" and the Demand for Heterogeneous Labour: Elasticity Estimates from a Multi-Factor Labour Demand Model for Germany, Applied Economics Letters 17(12), 2010, S. 1177- 1182 
Hagist, C., B. Raffelhüschen (2003): Generationen- vs. Bürgerversicherung: Welches Modell steht für mehr Nachhaltigkeit in der GKV? https://www.allianz.com/staticresources/images/pdf/saobj_222486_gutachten_raffelh_schen.pdf.

Houben, H., R. Maiterth, H. Müller (2011), Aufkommens- und Verteilungsfolgen des Ersatzes des deutschen einkommensteuerlichen Formeltarifs durch einen Stufentarif, Perspektiven der Wirtschaftspolitik 12(3), S. 280-302.

Kirchhof, P. (2003): Einkommensteuergesetzbuch: Ein Vorschlag zur Reform der Einkommen- und Körperschaftsteuer, C.F. Müller Verlag, Heidelberg.

Kirchhof, P. (2011): Bundessteuergesetzbuch: Ein Reformentwurf zur Erneuerung des Steuerrechts, C.F. Müller Verlag, Heidelberg.

Landais, C., T. Piketty, E. Saez (2011): Pour une Révolution Fiscale: Un Impôt sur le Revenu pour le XXIème Siècle, Le Seuil, Paris.

Löffler, M., A. Peichl, N. Pestel, H. Schneider und S. Siegloch (2012): Einfach ist nicht immer gerecht: Eine Mikrosimulationsstudie der Kirchhof-Reform für die Einkommensteuer, Vierteljahreshefte zur Wirtschaftsforschung 80(4), S. 147-160, DIW Berlin.

Mitschke, J. (2004): Erneuerung des deutschen Einkommensteuerrechts. Gesetzestextentwurf und Begründung, Mit einer Grundsicherungsvariante. Köln.

Neumann, D., A. Peichl, H. Schneider, S. Siegloch (2009): Die Steuerreformpläne der neuen Bundesregierung und das Bürgergeld: Eine Simulation von Risiken und Nebenwirkungen, Wirtschaftsdienst 89(12), 2009, S. 805-812.

OECD (2011): Taxing Wages 2009-2010, Paris.

Peichl, A. und T. Schaefer (2009): Steuerprogression in Europa: Eine Simulationsanalyse mit EUROMOD, in Habla, H. and Houben, H. (Hrsg.) Forschung mit Daten der amtlichen Statistik in Niedersachsen (FoDaSt), Statistik und Wissenschaft, Band 12, Statistisches Bundesamt, Wiesbaden.

Peichl, A. und S. Siegloch (2012): Accounting for Labor Demand Effects in Structural Labor Supply Models, Labour Economics, 19 (1), S. 129-138.

Peichl, A., N. Pestel, H. Schneider, S. Siegloch (2010): Alter Wein in neuen Schläuchen: Der Fünf-Stufen-Steuertarif der FDP auf dem Prüfstein, IZA Standpunkte, Nr. 27. 
Peichl, A., N. Pestel, H. Schneider, S. Siegloch (2011): Bemessungsgrundlage kontra Fünf-Stufen-Tarif: Eine Simulationsanalyse des Reformvorschlags nach Rose, Wirtschaftsdienst 91(5), S. 328-332.

Rose, M. (2011): Vorschlag für eine Reform des Einkommensteuertarifs, Wirtschaftsdienst 91(5), S. 323-327.

Rothgang, H., R. Arnold, R. Unger (2010): Berechnungen der finanziellen Wirkungen verschiedener Varianten einer Bürgerversicherung in der Gesetzlichen Krankenversicherung. Gutachten im Auftrag der Bundestagsfraktion Bündnis 90/Die Grünen. http://www.gruenebundestag.de/cms/gesundheit/dokbin/357/357085.gutachten_buergerversicherung.pdf RWI/FiFo Köln (2007): Der Zusammenhang zwischen Steuerlast- und Einkommensverteilung, Rheinisch-Westfälisches Institut für Wirtschaftsforschung und Finanzwissenschaftliches Forschungsinstitut an der Universität zu Köln, Forschungsprojekt für das Bundesministerium für Arbeit und Soziales.

RWI/FiFo Köln (2009), Wer trägt den Staat? - Die aktuelle Verteilung von Steuerund Beitragslasten auf die Bevölkerung in Deutschland, RWI Projektberichte. Essen.

RWI (2010), Varianten für einen Einkommensteuertarif 2011, RWI Projektberichte. Essen.

RWI (2011), Wer trägt den Staat? RWI Positionen \#43. Essen.

Slemrod, J. (1996): High-Income Families and the Tax Changes of the 1980s: The Anatomy of Behavioral Response, in: M. Feldstein and J. Poterba (Hrsg.), Empirical Foundations of Household Taxation, University of Chicago Press, S. 169-192.

Sozio-oekonomisches Panel (SOEP) (2009): Daten für die Jahre 1984-2009, Version 26, SOEP 2009.

van Soest, A. (1995): Structural Models of Family Labor Supply: A Discrete Choice Approach, Journal of Human Resources 30(1), S. 63-88.

Wissenschaftlicher Beirat beim Bundesministerium der Finanzen (2008): Existenzsicherung und Erwerbsanreiz, Gutachten des Wissenschaftlichen Beirats beim Bundesministerium der Finanzen, Berlin. 